\title{
Software Simulation Method for Digital IF GNSS Signal
}

\author{
Xiangyu Wu, Jinrui Liu, Hang Gong, Gang Ou \\ Satellite Navigation R\&D Center, National Univ. of Defense Technology, China \\ wxy8203@yahoo.com.cn
}

\begin{abstract}
Data simulation is an important auxiliary method for baseband signal processing algorithm design of digital IF GNSS receiver. Especially when the high sensitivity and high dynamic receivers are developed, or multipath and cross correlation mitigation techniques are considered, exact and complete tests under all kinds of necessary scenes can be carried out with the IF signal data which is generated by software simulation method. With this method, the developing efficiency can be improved and the cost can be cut down. In this paper, precise model of the IF signal is given firstly through analyzing GNSS receivers' RF processing flow. Then, considering the discrete characteristic of software processing, the IF signal simulation flow and block of the simulation software are designed. Finally, the specific design process is given through a design example. When the IF signal simulation software is used for testing baseband signal processing algorithms, GNSS receivers' IF signal data under every necessary test scene can be generated just by parameter adjustment.
\end{abstract}

Keywords-GNSS receiver; Digital IF signal; Software simulation; Spectrum aliasing; Band limiting filter

\section{INTRODUCTION}

In the signal processing flow of intermediate frequency (IF) GNSS receiver, the signal acquisition and tracking performance determines the precision of pseudo-range directly, and it is an important factor which affects the positioning, speed measuring and timing performance [1][2]. Baseband signal processing algorithms for high sensitivity, high dynamic receiver and multipath, cross correlation mitigation techniques have gained much attention in the field of high performance GNSS receivers' research [3]-[6]. Software simulation is an important auxiliary method in baseband signal processing algorithms' developing. It uses computer to implement all (or part of) the functions of baseband signal processing, so both the signal parameter adjustment and the statistical analysis of simulation results can be carried out very conveniently.

The software simulation method is much cheaper than the special IF signal collection hardware equipment. Besides, this method can evaluate the frequency and phase tracking performance and BER of navigation data bits conveniently, so it is more practical and effective. Common signal processing algorithms often ignore the code Doppler frequency, the influence of IF filter and the sampling effect. But these factors can influence high sensitivity, high dynamic signal processing and multipath, cross correlation mitigation algorithms sensitively. This paper focuses on the software simulation of GNSS receivers' IF signal. Processing flow and block of the simulation software is given, and it is illustrated concretely through a design example.

\section{IF SIGNAL MODEL}

The structure of GNSS receiver is illustrated in Fig. 1. The received signal is amplified by the preamplifier, and then mixed with the signal generated by local oscillator. After filtered by the band pass filter, the received signal is converted to IF. The bandwidth of the band pass filter is determined by requirement of the baseband signal processing algorithms. The output of the A/D converter is feed to digital baseband signal processing unit, in which the navigation data is demodulated and the pseudo-range, pseudo-range rate measurements are taken through signal acquisition and tracking process. At last, the position, velocity and time (PVT) determination and navigation filtering are implemented in a microprocessor generally.

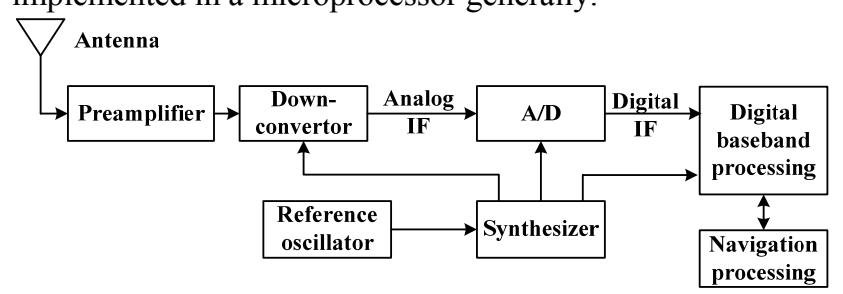

Figure 1. Top block diagram of digital IF GNSS receiver

All necessary clock signals are derived from the reference oscillator by the frequency synthesizer, base on the frequency plan of the receiver design. Because the reference oscillator has frequency error $\delta f_{r}(t)$, the local oscillator and sampling signal also have frequency error denoted as $\delta f_{L O}(t)$ and $\delta f_{S A}(t)$ separately. The mathematic model of the RF front end output analog IF signal is

$$
\begin{aligned}
r(t)= & \sum_{i=1}^{N} A_{i} \cdot D_{i}\left(t+F_{d i}(t) / f_{T}-\tau_{D i}\right) \\
& C_{i}\left(t+F_{d i}(t) / f_{T}-\tau_{i}\right) \cdot \cos \left(2 \pi f_{I F} t+\theta_{\Delta i}(t)+\theta_{i}\right) \\
& +n(t)
\end{aligned}
$$

where $A_{i}$ is the signal amplitude, $D_{i}(1$ or -1$)$ is the navigation data bit. $C_{i}$ is the pseudo random noise (prn) code, $f_{T}$ is the nominal frequency of transmitted carrier by satellites, $\tau_{D i}$ and $\tau_{i}$ are the phase delay of data bits and prn code respectively. $F_{d i}(t)$ is the integration of Doppler frequency $f_{d i}(t)$, and 
$F_{d i}(t) / f_{T}$ denotes the Doppler effect influenced on navigation data rate and prn code rate. $f_{I F}$ is the receiver's nominal IF frequency. $\theta_{\Delta i}(t)$ is the carrier phase offset introduced by the carrier frequency error $f_{\Delta i}(t)\left(=f_{d i}(t)-\delta f_{L O}(t)\right)$, and $\theta_{i}$ is the initial phase of the received carrier. The first item of the above equation is summation of $N$ received satellites' signal. $n(t)$ is zero mean Gaussian white noise with two sides spectrum power density $N_{0} / 2$ bandwidth $2 B$, which is centered at the frequency $f_{I F}$.

The carrier-to-noise power ratio (CNR) is an evaluation of the signal quality degradation due to noise. RF front end noise figure $(N F)$ is used to describe the degradation by $\mathrm{RF}$ processing. When the room temperature equals to $290 \mathrm{~K}$, the IF noise spectrum power density is $N_{0}=K_{B} T_{0} N F=-204$ $\mathrm{dBW} / \mathrm{Hz}+N F \mathrm{~dB}$. The definition of CNR is

$$
C N R=A^{2} / 2 N_{0}
$$

The sampling frequency must satisfy the band pass sampling theorem, and should be determined in the frequency plan, considering design requirements of RF signal down conversion, the choice of $f_{I F}$, design of frequency synthesizer and baseband algorithms [8]. The processing loss caused by quantification is related to the IF signal bandwidth, bit width and threshold setting of the quantification. The research conclusions about this question are given in [9], so the non-quantified discrete IF signals generated by software can be used for simulating baseband signal processing algorithms directly, and the quantification loss should be considered at last.

\section{Flow AND Structure OF SOFtware Simulation}

The flow of IF signal simulation is similar to the navigation signal generation on satellite [7]. Firstly, the navigation data bits are spread by prn code. Secondly, the spread signal is filtered by a band pass filter to limit its bandwidth. Then the filtered signal is modulated by carrier. More detailed processing flow and structure are determined by the values of the parameters in the signal model and their relationship. The nominal IF frequency $f_{I F}$, the IF signal bandwidth $2 B$ and the nominal sampling frequency $f_{\mathrm{s}}$ are constant, and they are determined form the frequency plan. The influence of reference oscillator frequency error on the IF carrier frequency offset and phase of each sample point is determined by the relation between reference oscillator, local oscillator and sampling clock frequency. Data bit $D_{i}$ and prn code $C_{i}$ should be consistent with the satellite number. Signal amplitude $A_{i}$ and noise power spectrum density $N_{0}$ are determined by CNR. The phase delay parameters $\tau_{D i}, \tau_{i}$ 、 $\theta_{i}$ can be set arbitrarily, but the data bit edge should be aligned with the prn code edge. The Doppler frequency $f_{d i}(t)$ can adopt a first or second order movement model.

In the receiver's RF processing, the analog IF signal is filtered by the anti-aliasing filter before $\mathrm{A} / \mathrm{D}$ conversion. The software generated prn code sequence has infinite bandwidth and the software processing contains sampling process, so the spectrum aliasing is inevitable. To mitigate spectrum aliasing, high sampling frequency must be adopted when generating prn code sequence. Single side bandwidth of the spread navigation data with high sampling frequency $F_{s}$ is always much larger than $f_{I F}$. Once the IF carrier modulation is performed directly, serious aliasing between the upper and down side band spectrum of the modulated signal will occur, and the quality of the simulated signal will degrade. So before the IF carrier modulation, it is necessary to filter the spread navigation data with a FIR low pass filter. The bandwidth of the low pass filter should be lower than $f_{I F}$ but larger than the single side bandwidth of IF signal, consequently the pass band of the signal modulated by IF carrier is not sensitive to the carrier Doppler frequency change. The noise generating process is similar to the IF signal's described above. The output of a Gaussian random number generator is filtered by FIR low pass filter and then modulated by IF carrier. Sum of the generated IF signal and noise is feed to a band pass filter with bandwidth $2 B$. The output of the filter is down sampled through decimating at last, in order to keep the generated signal rate equal to $f_{s}$.

According to the above analysis, the flow and software structure of IF signal simulation is illustrated in Fig. 2. The data bits of each satellite can be simulated with random binary sequence, or a piece of real navigation data demodulated from received satellite signal can be used. For signals with short prn code period, the edges of navigation data bits are aligned with the prn code period, no matter how the Doppler frequency change. So accurate navigation data phase control can be obtained easily by using prn code cycle counter as its phase controller. Both the code and carrier $\mathrm{NCO}$ are driven by frequency $F_{s}$. Phase increment of adjacent points is determined by frequency of the signal which is to be generated.

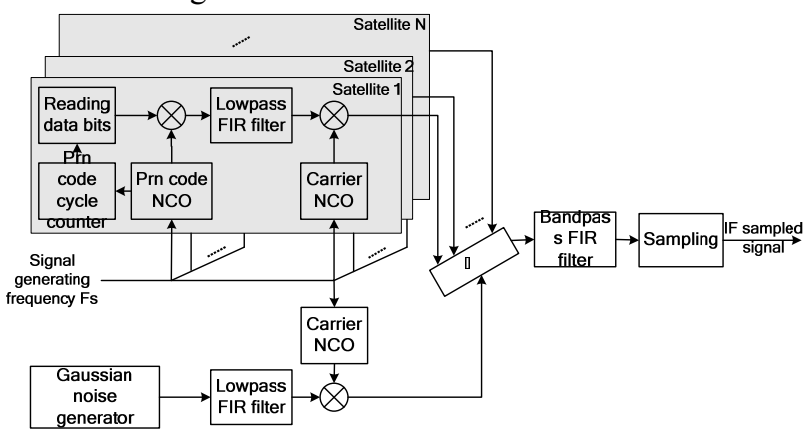

Figure 2. Flow diagram of software simulation for IF signal

\section{DESIGN EXAMPLE}

In this section, the GPS L1 C/A code IF discrete signal simulation is taken as an example to illustrate the calculation method of design parameters and the structure of each module in Figure 2.

\section{A. Determination of $f_{I F}, B$ and $f_{s}$}

$f_{I F}, B$ and $f_{s}$ are derived from the frequency plan considering the receiver's reference oscillator frequency and the requirement of RF, baseband processing. In this paper, the frequency plan of a typical GPS RF product is adopted. 
According the plan, nominal frequency of the reference oscillator is $f_{r}=16.367667 \mathrm{MHz}$, and local oscillator frequency $f_{L O}$ is 96 times of $f_{r} . f_{I F}$ is equal to $4123968 \mathrm{~Hz} . B$ is set to $1.023 \mathrm{MHz}$. Considering the band pass signal sampling theorem and design convenience of the frequency synthesizer, $f_{s}$ is set to $5455889 \mathrm{~Hz}$.

\section{B. Determination of $F_{s}, \sigma^{2}$ and $A$}

Spectrum aliasing is inevitable in generating sampled prn code sequence ( 1 or -1$)$ by software. To mitigate the aliasing, prn code sequence is firstly generated with high sampling frequency $F_{s}$, which is 20 times of the prn code rate. It is ensured that the main lobe of the spectrum do not alias with the first to ninth lobe. Secondly the sequence is filtered by low pass filter to restrict the bandwidth, and then output of the filter is decimated to the IF sampling frequency. For convenient sampling, $F_{s}$ can be set as 4 times of the real IF sampling frequency. If the real frequency of the receiver's oscillator is $9 \mathrm{~Hz}$ smaller than its nominal value, $F_{s}$ should be set to $4\left(f_{s}-3\right)=21823544 \mathrm{~Hz}$. This value is slightly larger than 20 times of the prn code rate, and it can simplify the decimation.

When zero-mean Gaussian noise is generated by random number generator, the noise variance $\sigma^{2}$ is the input parameter. Because the noise bandwidth is half of the sampling rate $F_{s}$, its single side power spectral density is $2 \sigma^{2} / F_{s}$. After modulated by IF carrier with unit amplitude, power spectral density of the IF noise becomes $N_{0}=\sigma^{2} / 2 F_{s}$. Thus the IF signal amplitude can be derived from the CNR definition as follow

$$
A=\sqrt{10^{C N R / 10} \sigma^{2} / F_{s}}
$$

\section{Carrier and Code NCO Control}

Driving clock frequency $F_{s}$, phase increment $N C O$ delta and the width of the phase accumulator $N$ are three basic parameters of NCO. The relationship between them and the generating signal frequency $f$ is ${ }^{[8]}$

$$
N C O \_ \text {delta }=2^{N} f / F_{s}
$$

The code frequency is $\left(1023000+f_{d i}(t) / 1540\right) \mathrm{Hz}$. Obviously, according to equation (4), real-time control of the generating code rate can be achieved based on the carrier Doppler frequency model. Phase delay $\tau_{i}$ corresponds to the initial value of the phase accumulation register in code NCO. One bit navigation data contains 20 code cycles. When cycle counter is used to control the scheduling of the navigation data, its Doppler characteristic will be consistent with the code's, and the bit boundary can always be aligned with a code periodic boundary. The sub-millisecond part of phase delay $\tau_{D i}$ corresponds to the code phase delay $\tau_{i}$, and its millisecond part is implemented by setting the code cycle counter to the corresponding initial value. The carrier frequency of the IF signal is $f_{I F}+f_{d i}(t)-\delta f_{L O}(t)$. The local oscillator frequency error $\delta f_{L O}(t)$ can be obtained by multiplying the reference frequency error with the ratio $f_{L O} /$ $f_{r}$. The initial carrier phase can be set arbitrarily. Frequency of the carrier used for modulating noise is $f_{I F}$.

\section{FIR Low PassFilter Design}

Carrier Doppler frequency of the received signal is usually within $-5 \mathrm{kHz} \sim 5 \mathrm{kHz}$, so the cutoff frequency of its pass band can be set to $1.5 \mathrm{MHz}$ according to the former discussed design principles of low pass filter bandwidth. Linear phase FIR low pass filter can be designed by window function method [10], and the Hann window with minimum stop band attenuation of- $44 \mathrm{~dB}$ is used. To ensure the steepness of the transition zone, the filter is set to 200th order, and the transition bandwidth is $3.1 \times F_{s} / 200=338265 \mathrm{~Hz}$. Figure 3 shows the amplitude and phase-frequency response curve of the FIR low pass filter within the frequency band of $0 \sim$ 2MHz.
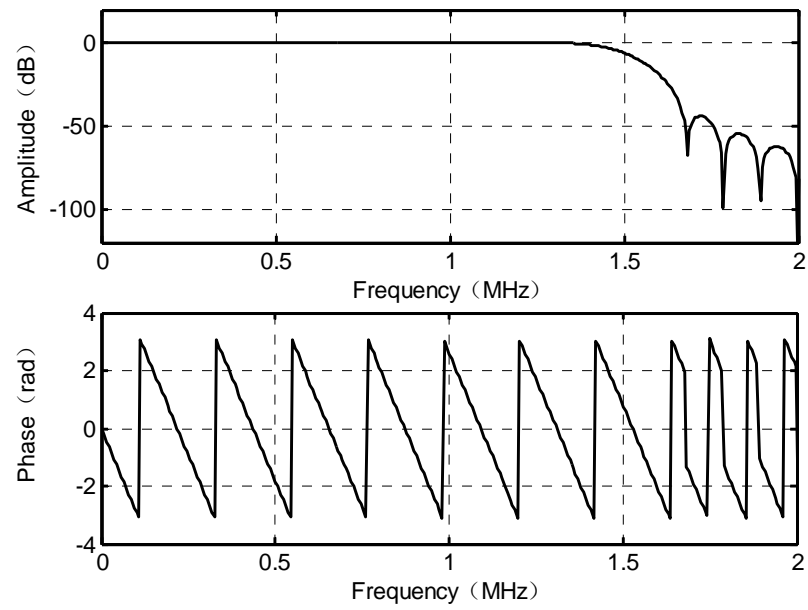

Figure 3. Amplitude and phase-frequency curves of FIR LPF

\section{E. FIR Band Pass Filter Design}

The FIR band pass filter before the decimator is used to simulate the anti-aliasing filter before $\mathrm{A} / \mathrm{D}$ conversion in receiver. Its central frequency is $f_{I F}$, and bandwidth is $2 B$. The linear phase FIR band pass filter can also be designed using Hann window function. Its order is set to 200, and cutoff frequency is set to $f_{I F}+B+3.1 \times F_{s} / 400=5.3161 \mathrm{MHz}$ and $f_{I F}-B-3.1 \times F_{s} / 400=2.9318 \mathrm{MHz}$. Figure 4 shows the amplitude and phase-frequency response curve of the filter within the frequency band of $0 \sim 8 \mathrm{MHz}$.

The final generated IF sampling signal is the output of the 1/4 decimator. The IF signal generated by software simulation and its spectrum are shown in Figure 5 (a) and (b) separately, where $\sigma^{2}=10000$ and $C N F_{I F}=45 \mathrm{dBHz}$. The sampling rate of the final generated signal is $F_{s} / 4=5455886$ $\mathrm{Hz}$. Central frequency of the signal is $f_{I F}$, but due to spectrum shift caused by the decimation the signal's spectrum band center in Figure 5 appeared at $\pm\left(F_{s} / 4-f_{I F}\right)= \pm 1331918$ $\mathrm{Hz}$. 

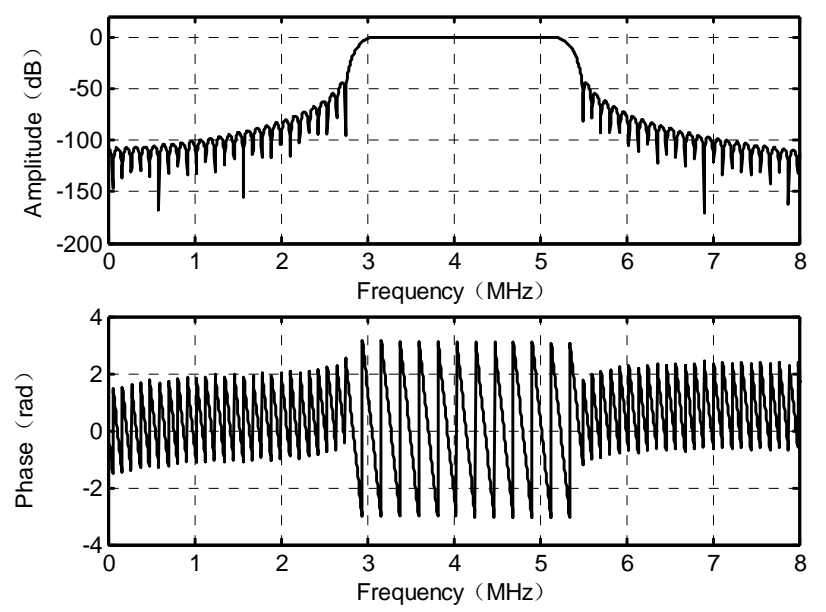

Figure 4. Amplitude and phase-frequency curves of FIR BPF

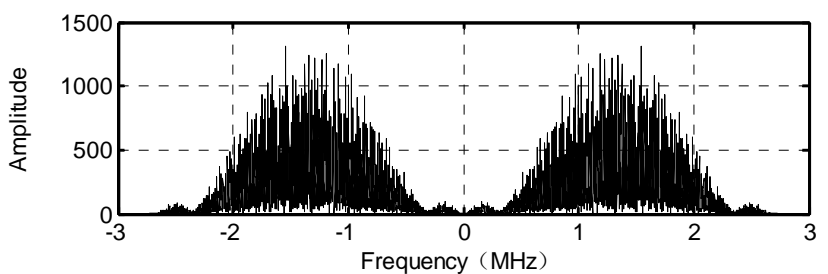

(a) IF signal spectrum

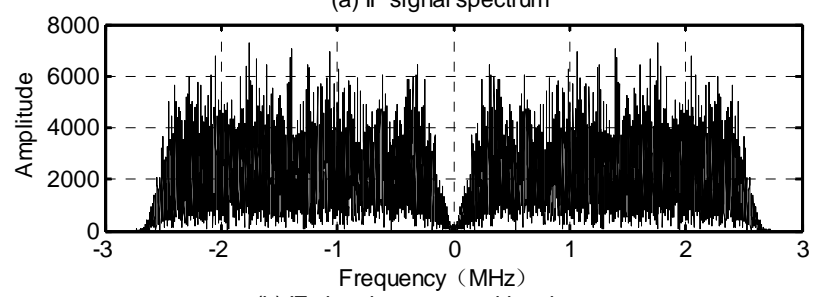

(b) IF signal spectrum with noise

Figure 5. Frequency spectrum of software simulated IF signal

\section{CONCLUSIONS}

The precise mathematical model for IF signals is established based on the analysis of the RF processing for the digital IF GNSS receiver. The flow for IF signal simulation with software is designed, regarding the relationship among the parameters in the established model and the characteristics of digital processing. The detailed design method of the software modules are also demonstrated. The time domain and frequency domain characteristics are heavily investigated in the simulation method design. With a few modifications of the related parameters, the simulation method can be applied to most baseband signal processing algorithms.

When dealing with the more complicated processing algorithm, considering the frequencies and phases relationship among different satellites and the similarity between some sub-frames of the navigation message, it is demanded to generate the navigation message、 phase delay and Doppler frequency shift precisely based on the location motion and signal arrival time of the receiver in addition to the satellite constellation orbit model. This will be the key research point on satellite constellation emulator in the next step.

\section{REFERENCES}

[1] B.Y. Tsui, Fundamentals of Global Positioning System Receivers: A Software Approach. Second Edition, Hoboken, New Jersey: John Wiley\&Sons, Inc., 2005.

[2] P. Misra, P. Enge, Global Positioning System Signals, Measurement, and Performance, Second Edition, Ganga-Jamuna Press. 2006.

[3] I.Z. Nessreen, GNSS Receivers for Weak Signals, Norwood, Massachusetts: Artech House, Inc., 2006.

[4] C.X. Li, F.X. Wang, "Correlation of PN spread spectrum signal under first-order dynamics," Acata Electronica Sinica, Beijing, vol. 35, No. 9, pp. 1769-1793, 2007. (in Chinese).

[5] H.C. Liu, Research on the Multipath Effects and Mitigation Techniques in Satellite Navigation Systems, National University of Defense Technology Doctor Dissertation, 2010,4. (in Chinese).

[6] E.P. Glennon, "A Review of GPS Cross Correlation Mitigation Techniques," The 2004 International Symposium on GNSS/GPS, Sydney, Australia, 6-8 December 2004.

[7] E.D. Kaplan, C.J. Hegarty, Understanding GPS Principles and Applications. Second Editon, Norwood, Massachusetts: Artech House, Inc., 2006.

[8] B.Y. Li, Y.H. Zhang, O. Gang, F.X. Wang, "Frequency planning for all digital receivers with given bandwidth," Signal Processing, Beijing, vol.24, No. 1, pp. 115-117, 2008. (in Chinese).

[9] A. J. Van Dierendonck, "GPS receivers," in Global Positioning System: Theory and Applications. vol. I, B.W. Parkinson, J.J. Jr.Spilker, eds. Washington: AIAA, 1996.

[10] P.Q. Cheng, Digital Signal Processing. Beijing: Tsinghua University Press. 2002. (in Chinese). 